\title{
Geometric and electronic structures of two-dimensionally polymerized triptycene: Covalent honeycomb networks comprising triptycene and polyphenyl
}

\author{
Yasumaru Fujii*, Mina Maruyama, and Susumu Okada \\ Graduate School of Pure and Applied Science, University of Tsukuba, 1-1-1 Tennodai, \\ Tsukuba 305-8571, Japan \\ *E-mail: yfujii@comas.frsc.tsukuba.ac.jp
}

Based on the density functional theory with the generalized approximation, we investigated the geometric and electronic structure of two-dimensional covalent networks consisting of triptycene and phenyl groups, which are alternately arranged in a hexagonal manner. Calculated total energies of the networks are a few tens $\mathrm{meV}$ per atom higher than that of an isolated benzene, indicating that the networks are energetically stable. All networks were semiconductors with a moderate band gap at the $\Gamma$ point, value of which inversely proportional to the length of polyphenyl connecting the triptycene. According to a kagome topology of $\pi$ electrons distributed on the $\mathrm{sp}^{2}$ hydrocarobons, the characteristic kagome energy bands consisting of a flat dispersion band and a Dirac cone emerge in valence and conduction states of which structure is sensitive to the mutual orientation of phenyl groups with respect to the polymer chain. 


\section{Introduction}

Electronic structure of nanoscale materials is characterized by their size, dimensionality, network topologies, and boundary conditions. Nanoscale carbon materials, such as aromatic hydrocarbons ${ }^{1)}$, fullerenes ${ }^{2)}$, and carbon nanotubes ${ }^{3)}$, are representative examples of such nanoscale materials. Aromatic hydrocarbons having primarily armchair (cis) edges possess basically symmetric electronic structure with respect to the Fermi level and a moderate energy gap between the highest occupied and the lowest unoccupied states. This gap is inversely proportional to the molecular size ${ }^{1)}$. In contrast, introducing an imbalance between the numbers of two sublattices in hexagonal networks creates the non-bonding states at the Fermi level, the number of which is the identical to that of the sublattice imbalance, leading to the spin polarization. ${ }^{4-9)}$ Polymerization of the hydrocarbons causes further variation in the resultant two-dimensional networks. ${ }^{10,11)}$ Their electronic properties depend on the constituent molecule and on the interconnect geometries. For example, the networks consisting of phenalenyl and phenyl groups possess Dirac cones arising from the hexagonally arranged phenalenyl and kagome bands owing to the phenyl interconnects which form the kagome lattice, even though the $\pi$ electrons are extended throughout the networks. Besides the networks comprising solely hexagonal rings, topological defects such as pentagonal and heptagonal rings causes unusual electronic structure being different from that of the conventional hexagonal networks. ${ }^{12,13)}$

Hybrid networks of $\mathrm{sp}^{2}$ and $\mathrm{sp}^{3} \mathrm{C}$ atoms show further variations in their geometric and electronic structures which do not appear in $\mathrm{sp}^{2} \mathrm{C}$ materials. Lacking $\pi$ electrons, $\mathrm{sp}^{3} \mathrm{C}$ atoms act as spacers for $\pi$ electron networks while giving structural flexibility, allowing the materials to form two- and three-dimensional structures. Iptycene is an example of such $\mathrm{sp}^{2}-\mathrm{sp}^{3}$ hybrid carbon molecule, which possesses a Y-shaped ridged structure consisting of three acene panels connected via bridgehead $\mathrm{sp}^{3} \mathrm{C}$ atoms with fourfold coordination situated at the molecular axis. ${ }^{14,15)}$ Because of the substantial wave function overlap among three acene panels, the electronic structure of the iptycene is slightly modulated from that of the constituent acene units, even though the acene panels are separated by $\mathrm{sp}^{3} \mathrm{C}$ atoms. By analogy with the covalent organic frameworks consisting of conventional 2D hydrocarbons, iptycene also can form covalent networks under the polymerization between 
adjacent acene units. Indeed, triptycene ${ }^{16-18)}$, consisting of two $\mathrm{sp}^{3} \mathrm{C}$ atoms and three benzene panels, possess polymeric networks. ${ }^{19-24)}$ In such networks, because the $\mathrm{sp}^{2}$ moiety of the triptycene polymer is arranged in the kagome topology, the electronic structure near the Fermi level exhibits unusual feature that are absent in the $\mathrm{sp}^{2} \mathrm{C}$ network materials. Indeed, our previous calculations indicated that networks comprising $\mathrm{sp}^{3} \mathrm{C}$ atoms and oligoacene are semiconductors with the peculiar kagome bands in their valence and conductions states. ${ }^{25,26)}$

In this work, following the synthesis of the triptycene polymers containing phenyl groups, we aim to investigate the energetics and electronic structure of the triptycene polymers in terms of the phenyl conformations and length, using the density functional theory (DFT) with the generalized gradient approximation (GGA). Our calculation showed that the triptycene polymers are energetically stable with the similar total energy to that of an isolated benzene. The polymers are semiconductors with the direct band gap between dispersive valence band and the flat conduction band at $\Gamma$ point with the band gap of about $2 \mathrm{eV}$ which is inversely proportional to phenyl length. The polymers possess peculiar electronic band structures in both valence and conduction states, originated from the kagome topology of the $\mathrm{sp}^{2}$ hydrocarbon networks.

\section{Calculation Method and Structural Model}

All calculations were conducted using the $\mathrm{DFT}^{27,28)}$ as implemented in the program package STATE. ${ }^{29)}$ We used GGA with the Perdew-Burke-Ernzerhof functional to describe the exchange-correlation potential energy among interacting electrons. ${ }^{30)}$ Ultrasoft pseudopotentials generated by the Vanderbilt scheme were adopted for describing the interaction between electrons and ions. ${ }^{31)}$ Valence wave function and deficit charge density were expanded in terms of plane-wave basis sets with cutoff energies of 25 and $225 \mathrm{Ry}$, respectively, which give sufficient convergence in the total energy and electronic structures of covalent networks consisting of both $\mathrm{sp}^{2}$ and $\mathrm{sp}^{3} \mathrm{C}$ atoms. ${ }^{32,33)}$ Molecular dynamics simulations were conducted using velocity scaling method to keep the temperature constant during the simulation to insure the thermal stability of the triptycene polymers. Integrations over the hexagonal Brillouin zones were excused by using equidistant $2 \times 2 \times 1$ $\boldsymbol{k}$-mesh. Lattice parameters and internal atomic structures were fully optimized until the 
force acting on each atom was less than $5 \mathrm{mRy} / \mathrm{A}$. To simulate an isolated two-dimensional covalent network, the network was separated from its periodic images by $10 \AA$ or $20 \AA$ vacuum spacing, depending on the thickness of the networks. For the network consisting only of triptycene, the vacuum spacing of $10 \AA$ was adopted, while the networks containing phenyl groups, $20 \AA$-vacuum spacing adopted.

Figure 1 shows an optimized structure of triptycene polymers. In this work, we consider three representative polymers in which hexagonally arranged triptycenes are connected directly [Fig. 1(a)], via a phenyl [Fig. 1(b)], and via a biphenyl [Fig. 1(c)] with three adjacent triptycenes. The model considered here qualitatively simulates the experimentally synthesized polymers and may provide the fundamental properties in terms of the length of phenyl interconnects, although the covalent network between adjacent triptycene is not exactly the same as the experimental one. With the choice of the structural models, the polymers are hybrid covalent networks of $\mathrm{sp}^{3}$ and $\mathrm{sp}^{2} \mathrm{C}$ atoms, which are arranged hexagonal manner as vertexes of the network and are arranged the kagome topology as the connecting units, respectively. Therefore, the $\pi$ electron systems satisfied the kagome network topology with an internal degree of freedom connected with its adjacent units via next nearest electron transfer around the $\mathrm{sp}^{3} \mathrm{C}$ atoms.

\section{Results and discussion}

Optimized lateral lattice parameters of polymerized triptycene are $15.38 \AA$ for the network without phenyl group (tp-tp) and 22.17 and $28.75 \AA$ for the networks containing phenyl (tp-ph-tp) and biphenyl (tp-bp-tp), respectively. Under the optimum lattice parameters, the optimized bond lengths of the polymers are summarized in Table I. The optimized bond length of the bridgehead $\mathrm{sp}^{3} \mathrm{C}$ atoms is $1.52 \AA$ which is the same as that of the isolated triptycene molecule. Furthermore, the bond length associated with the $\mathrm{sp}^{2} \mathrm{C}$ are about $1.40 \AA$ and found to be insensitive to polymerization for all polymers. Thus, the polymerization does not substantial structural modification in triptycene unit. By focusing on the bond connecting triptycene units with the other triptycene or phenyl groups, we found that the bond $\mathrm{d} 9$ indicate the single bond nature because of the slightly long bond length of $1.48 \AA$. In addition, the bond connecting two phenyl units in the structure tp-bp-tp is also longer than the bond forming benzene rings in phenyl and triptycene, 
indicating its single bond nature. As for the bond in benzene rings in phenyl groups, the optimized bond lengths are also about $1.4 \AA$, as the case of the conventional $\mathrm{sp}^{2} \mathrm{C}$ materials.

Figure 2 shows the total energy per $\mathrm{C}$ atom of triptycene polymers as a function of the lattice constant, with respect to the energy of an isolated benzene. The total energy monotonically decreases with increasing the lattice parameter or the phenyl unit length. The calculated total energies are 63, 52, and $48 \mathrm{meV} /$ atom for the networks of tp-tp, tp-ph-tp, and tp-bp-tp, respectively, with respect to the energy of the benzene, indicating that all networks are energetically stable. The decrease of the total energy is ascribed to the substantial decrease of the kinetic energy of the polymer with the increase of the phenyl length, although the Coulomb repulsive interaction between $\mathrm{H}$ atoms attached to triptycene and phenyl increase the total energy with increasing the phenyl length.

The dynamical stability of the triptycene polymers was also investigated by ab-initio molecular dynamics simulations conducted at a constant temperature up to $1500 \mathrm{~K}$ for simulation times of 0.2 ps. Under the elevated temperature, all structures retained their initial network topologies for the above simulation times. Therefore, the polymers are also dynamically stable at the ambient conditions.

Figure 3 shows the electronic structure of polymeric triptycene with the structures of tp-tp, tp-ph-tp, and tp-bp-tp. All polymers were semiconductors with the direct band gap at the $\Gamma$ point, width of which depends on the phenyl length. In addition, the polymers possess peculiar band structures in both valence and conduction stats: The most of the electron states show the kagome band feature consisting of three branches where two of three have substantial dispersion forming a Dirac cone at the $\mathrm{K}$ point while the remaining state exhibited a flat band nature throughout the Brillouin zone. According to the kagome band structure, the band edge exhibit unusual feature: the lowest branch of the conduction band and the third highest branch of the valence band exhibit perfect flat band nature, indicating the possibility of the strong adsorption between these two states. In addition, despite the $\pi$ electron network is segmented in each panel consisting of benzene rings and terminated by 
$\mathrm{sp}^{3} \mathrm{C}$ atoms situated at the network vertexes, each branch of the electronic energy bands possesses substantial bandwidth except the branches associated with the kagome flat band. The calculated bandwidth is about $0.5 \mathrm{eV}$ or larger depending on the branches and phenyl length. This indicates that $\pi$ electrons in the triptycene polymers are extended throughout the covalent framework with the spatially dependent electron hopping among the $\mathrm{sp}^{2} \mathrm{C}$ atomic sites, even though they are segmented by $\mathrm{sp}^{3} \mathrm{C}$ atoms. Indeed, the squared wave function of the flat band state extended throughout the network with $\pi$ electron nature.

To provide further insight into the electronic band structure of the triptycene polymers, we analyze the band gap [Fig. 4(a)], band width of the highest and the lowest three branches of the kagome bands [Fig. 4(b)], and the eigen value of the highest occupied and the lowest unoccupied flat bands [Fig. 4(c)]. The band gap monotonically decreases with increasing the phenyl length: The band gap of the tp-bp-tp polymer is narrower than that of the tp-tp polymer by $0.5 \mathrm{eV}$ [Fig. 4(a)]. The gradual decrease of the band gap is caused by the decrease of the bandwidth of the highest three occupied and the lowest three unoccupied states. Indeed, the bandwidth of these states decreases with the insertion of biphenyl into the pristine triptycene polymer or the tp-tp polymer by $0.5 \mathrm{eV}$, corresponding with the decrease of the band gap. The bandwidth decrease of these states also causes the upward shifts and the downward shifts of the kagome flat band states. Note that the bandwidth of the next highest branches of the valence band is insensitive to the length of phenyl groups inserted into triptycene vertexes. Thus, the decrease of the bandwidth of the highest and lowest branches is ascribed to the wave function nature with respect to the phenyl length. We investigated the effective masses of the valence and conduction bands. For the conduction band edge, the effective masses for the dispersive band are 1.8, 2.3, and $2.4 m_{e}$ for triptycene polymers with tp-tp, tp-ph-tp, and tp-bp-tp structures, respectively. In contrast, as for the flat band at the conduction band edge, we found that their effective electron mass is $30,000 m_{e}$ or heavier, indicating that the kagome flat band provide the heavy electron system. For the valence band edge, the hole effective masses are 2.0, 1.7, and $1.4 m_{e}$ for triptycene polymers with tp-tp, tp-ph-tp, and tp-bp-tp strictures, respectively.

Because of the steric hindrance of the $\mathrm{H}$ atoms in biphenyl, phenyl units prefer to rotate 
with respect to their polymeric axis to decrease Coulomb repulsive interaction between adjacent phenyl units. Therefore, the phenyl unit in the polymers can rotate with their polymer axes. Figure 5 shows the total energy of the polymers containing phenyl and biphenyl as a function of the rotational angle with respect to the polymer axes. As the case of the isolated polyphenyl molecule, the inserted phenyl group prefers the tilted conformation with the tilt angle of 35 degree than the networks with flat conformation. On the other hand, the angle of 90 degree is the lest stable, indicating that the $\pi$ electrons on the network itinerates throughout the covalent networks. Thus, the preferential angle is determined by competition between itineracy of $\pi$ electrons and the Coulomb repulsive interaction between $\mathrm{H}$ atoms attached to phenyl units.

Since the phenyl inserted into triptycene polymer prefers the tilted conformations, we investigate the electronic structure of triptycene polymer containing phenyl with tilted conformations (Fig. 6). The rotation of phenyl causes the upward and downward shifts of the three lowest unoccupied and the three highest occupied states, respectively [Fig. 6(a)]. According to the downward shift of the highest three occupied states, the electronic structure near the valence band edge is substantially modulated. At the rotation angle of 50 degree, the highest three occupied states loose their kagome band nature, instead the next highest three states exhibit the kagome band nature [Fig. 6(b)]. At the rotation angle of 90 degree, the highest two occupied states loose their band dispersion, indicating that $\pi$ electron transfer is terminated by the phenyl rotation [Fig. 6(c)]. Indeed, the wave function of the highest occupied state at the $\Gamma$ point is localized on the triptycene unit.

For the triptychene polymer containing biphenyl interconnects, the electronic structure exhibits different characteristics by the phenyl rotation (Fig. 7). The kagome band nature of the most of valence and conduction states disappears: The phenyl rotation makes a finite energy gap in the Dirac cone at the K point with the angle of 50 degree or larger [Fig. 7(b)]. At the angle of 90 degree, the most of valence and conduction states exhibits flat band nature indicating that the $\pi$ electron system is localized on the particular moiety of the network [Fig. 7(c)]. Indeed, wave function of the highest occupied state at the $\Gamma$ point is absent on one of two triptycene in the network, indicating their highly localized nature. 
Since the rotation of phenyl modulates the $\pi$ electron transfer between the adjacent $\mathrm{sp}^{2} \mathrm{C}$ region, we make qualitative discussion of the modulation of the kagome band with respect to the $\pi$ electron transfer, using the simple tight-binding model. We consider the structural model that linear chains consisting three states form the kagome lattice with the inter- and intra-chain electron transfer of $t$ and $t^{\prime}$, respectively, to simulate the triptycene polymer containing phenyl. By changing the intra-electron transfer $t$ corresponding with the phenyl rotation, the flat band state shifts downward from the dispersive band to the other one, as in the case of the electron states of the polymer with the phenyl rotation of 50 degree or larger [Fig. 8(a)]. Therefore, the substantial energy shift of the flat band in the polymer is ascribed to the modulation of electron transfer between inter- and intra-phenyl units. For the triptycene polymer containing biphenyl, we consider the structural model shown in Fig. 8(b): Linear atomic chains consisting 4 atomic sites are arrange in the kagome network in which two different intra-chain transfer, $t^{\prime}$ and $t^{\prime \prime}$, are taken into account under the fixed inter-chain transfer $t=0.5$. By changing the ratio of $t$ ' and $t^{\prime \prime}$, the dispersive zero gap band has a finite energy gap at the $\mathrm{K}$ point and loses its kagome band nature. Therefore, this concludes that the delicate balance among the electron transfer within $\mathrm{sp}^{2} \mathrm{C}$ moiety and between adjacent $\mathrm{sp}^{2}$ panel separated by $\mathrm{sp}^{3} \mathrm{C}$ atoms causes the peculiar electronic structure of the triptycene polymers.

\section{Conclusion}

Using DFT with GGA, we studied the geometric and electronic structures of triptycene polymers consisting of triptycene and phenyl groups, in terms of the phenyl group length inserted between triptycene units. Our calculation showed that triptycene polymers are energetically stable with the similar total energy to that of benzene. The polymers are semiconductors with the direct band gap between dispersive valence band and the flat conduction band at the $\Gamma$ point with the band gap of about $2.5 \mathrm{eV}$, which is inversely proportional to phenyl length. The polymers possess peculiar electronic band structures in both valence and conduction states, originated from the kagome topology of the $\mathrm{sp}^{2}$ hydrocarbon in the polymer. The wave function distribution of the flat band states indicates that the state extended throughout the hydrocarbon network, even though the $\mathrm{sp}^{2} \mathrm{C}$ 
moieties are separated by the $\mathrm{sp}^{3} \mathrm{C}$ atoms at the molecular axis of triptychene. This indicates that the triptychene polymers could be regarded as the two-dimensional $\mathrm{sp}^{2}$ kagome networks with internal degree of freedom. We also demonstrated that the electronic structure of the polymers is further modulated by rotating the phenyl groups inserted between triptycene units, changing the electron transfer within and between $\mathrm{sp}^{2} \mathrm{C}$ moieties.

\section{Acknowledgments}

The author thanks JST-CREST Grant Numbers JPMJCR1532 and JPMJCR1715 from the Japan Science and Technology Agency, JSPS KAKENHI Grant Numbers JP17H01069, JP16H00898, and JP16H06331 from the Japan Society for the Promotion of Science, and the Joint Research Program on Zero-Emission Energy Research, Institute of Advanced Energy, Kyoto University. Part of the calculations was performed on an NEC SX-Ace at the Cybermedia Center at Osaka University and on an SGI ICE XA/UV at the Institute of Solid State Physics, The University of Tokyo. 


\section{References}

1) E. Clar, The Aromatic Sextet (John Wiley and Sons, London, U.K. 1972).

2) H. W. Kroto, J. R. Heath, S. C. O’Brien, R. F. Curl, and R. E. Smalley, Nature 318, 162 (1985).

3) S. Iijima, Nature 354, 56 (1991).

4) Y. Morita, S. Suzuki, K. Sato, and T. Takui, Nature Chem. 3, 197 (2011).

5) V. Boekelheide and C. E. Larrabee, J. Am. Chem. Soc. 72, 1245 (1950).

6) D. H. Reid, Tetrahedron 3, 339 (1958).

7) K. Nakasuji, M. Yamaguchi, I. Murata, K. Yamaguchi, T. Fueno, H. Ohya-Nishiguchi, T. Sugano, and M. Kinoshita, J. Am. Chem. Soc. 111, 9265 (1989).

8) Z. Sun and J. Wu, J. Mater. Chem. 22, 4151 (2012).

9) T. Kubo, Chem. Rec. 15, 218 (2015).

10) M. Maruyama, N. T. Cuong, and S. Okada, Carbon 109, 755 (2016).

11) M. Maruyama and S. Okada, Carbon 125, 530 (2017).

12) K. Kusakabe, K. Wakabayashi, M. Igami, K. Nakada, and, Mol. Cryst. Liq. Cryst. 305, 445 (1997).

13) M. Maruyama and S. Okada, Appl. Phys. Express 6, 095101 (2013).

14) P. D. Barlett, M. J. Ryan, and S. G. Cohen, J. Am. Chem. Soc. 64, 2649 (1942).

15) H. Hart, S. Shamouilian, and Y. Takehira, J. Org. Chem. 46, 4427 (1981).

16) C. F. Chen, Chem. Commun. 47, 1674 (2011).

17) T. M. Swager, Acc. Chem. Res. 41, 1181 (2008).

18) M. Mastalerz and I. M. Oppel, Angew. Chem. Int. Ed. 51, 5252 (2012).

19) P. Kissel, D. J. Murray, W. J. Wulftange, V. J. Catalano, and B. T. King, Nature Chem. 6, 774 (2014).

20) M. Xue and C. F. Chen, Chem. Commun. 47, 2318 (2011).

21) R. Bhola, P. Payamyar, D. J. Murray, B. Kumar, A. J. Teator, M. U. Schmidt, S. M. Hammer, A. Saha, J. Sakamoto, A. D. Schluter, and B. T. King, J. Am. Chem. Soc. 135, 14134 (2013).

22) C. Zhang, P. C. Zhu, L. Tan, J. M. Liu, B. Tan, X. L. Yang, and H. B. Xu, Macromolecules, 48, 8509 (2015).

23) Q. Liang, S. Cui, S. Xu, C. Yao, M. J. MacLachlan, and Z. Li, Chem. Commum. 54, 3391 
(2018).

24) B. S. Ghanem, M. Hashem, K. D. M. Harris, K. J. Msayib, M. Xu, P. M. Budd, N. Chaukura, D. Book, S. Tedds, A. Walton, and N. B. McKeown, Macromolecules. 43, 5287 (2010).

25) J. Sorimachi and S. Okada, Phys. Rev. B 96, 024103 (2017).

26) Y. Fujii, M. Maruyama, K. Wakabayashi, K. Nakada, and S. Okada, J. Phys. Soc. Jpn. 87, 034704 (2018).

27) P. Hohenberg and W. Kohn, Phys. Rev. 136, B864 (1964).

28) W. Kohn and L. J. Sham, Phys. Rev. 140, A1133 (1965).

29) Y. Morikawa, K. Iwata, and K. Terakura, Appl. Surf. Sci. 169-170, 11 (2001).

30) J. P. Perdew, K. Burke, and M. Ernzerhof, Phys. Rev. Lett. 77, 3865 (1996).

31) D. Vanderbilt, Phys. Rev. B 41, 7892 (1990).

32) M. Maruyama, N. T. Coung, and S. Okada, J. Phys. Soc. Jpn. 84, 084706 (2015).

33) M. Maruyama and S. Okada, Diamond Relat. Mater. 81, 103 (2018). 


\section{Figure Captions}

Fig. 1. (Black and white) Geometric structure of polymerized triptycene with (a) tp-tp, (b) tp-ph-tp, and (c) tp-bp-tp structures. Black and white balls indicate C and $\mathrm{H}$ atoms, respectively.

Fig. 2. (Color Online) Total energy of the two-dimensional covalent networks consisting of triptycne and phenyl group as a function of the lattice constant. The energy is measured from that of the benzene.

Fig. 3. (Color Online) Electronic structure of the polymerized triptycene with (a) tp-tp, (b) tp-ph-tp, (c) tp-bp-tp structures. The vacuum level energy is set to zero. Arrows indicate the top of the valence bands.

Fig. 4. (Color Online) (a) The band gap of the polymerized triptycene, (b) the bandwidth of the kagome band near the Fermi level, and (c) the kagome flat band levels near the Fermi level as a function of the lattice constant. Triangles and rhombuses in 4(b) and 4(c) correspond with the energies of the valence and conduction bands, respectively.

Fig. 5. (Color Online) Total energy of the tp-ph-tp polymer and tp-bp-tp polymer as a function of the rotation angle of phenyl groups. Triangles and rhombuses denote the energies of the tp-ph-tp and tp-bp-tp polymers, respectively.

Fig. 6. (Color Online) Electronic structures of the triptycene polymer containing phenyl with its rotation angle of (a) 30, (b) 50, and (c) 90 degrees. Arrows indicate the top of the valence bands.

Fig. 7. (Color Online) Electronic structures of the triptycene polymer containing biphenyl with its rotation angle of (a) 30, (b) 50, and (c) 90 degrees. Arrows indicate the top of the valence bands. 
Fig. 8. (Color Online) Band structures of kagome networks of atomic chains consisting (a) three atomic sites and (b) four atomic sites, simulating the triptycene polymers containing phenyl and biphenyl, respectively. 
Table I. The bond length of fully optimized two-dimensional covalent networks of triptycene and phenyl groups. The bond labels are defined in Fig. 1(b).

\begin{tabular}{|c|c|c|c|c|c|c|c|c|c|c|c|c|c|c|c|c|}
\hline \multirow[t]{2}{*}{ metwork } & \multicolumn{16}{|c|}{ Bond Length $[\AA]$} \\
\hline & d1 & d2 & d3 & d4 & d5 & $\mathrm{d} 6$ & d7 & $\mathrm{d} 8$ & d9 & $\mathrm{d} 10$ & d11 & d12 & d13 & d14 & $d 15$ & d16 \\
\hline $\mathrm{tp}-\mathrm{tp}$ & 1.52 & 1.52 & 1.40 & 1.39 & 1.39 & 1.38 & 1.41 & 1.41 & 1.48 & - & - & - & - & - & - & - \\
\hline tp-ph-tp & 1.52 & 1.53 & 1.40 & 1.39 & 1.39 & 1.39 & 1.41 & 1.42 & 1.48 & 1.41 & 1.41 & 1.39 & 1.39 & - & - & - \\
\hline$t p-b p-t p$ & 1.52 & 1.53 & 1.40 & 1.39 & 1.39 & 1.39 & 1.41 & 1.41 & 1.48 & 1.41 & 1.41 & 1.39 & 1.39 & 1.41 & 1.41 & 1.48 \\
\hline
\end{tabular}


(a)
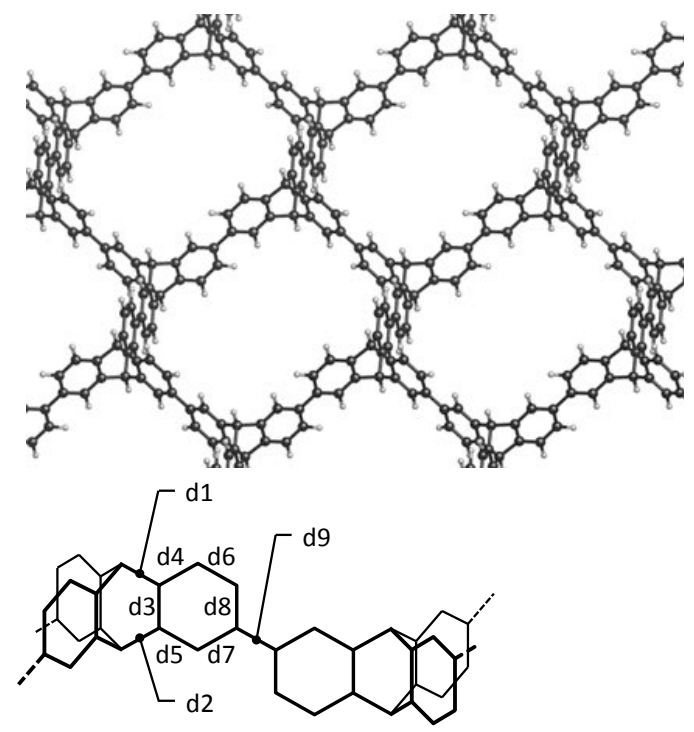

(b)

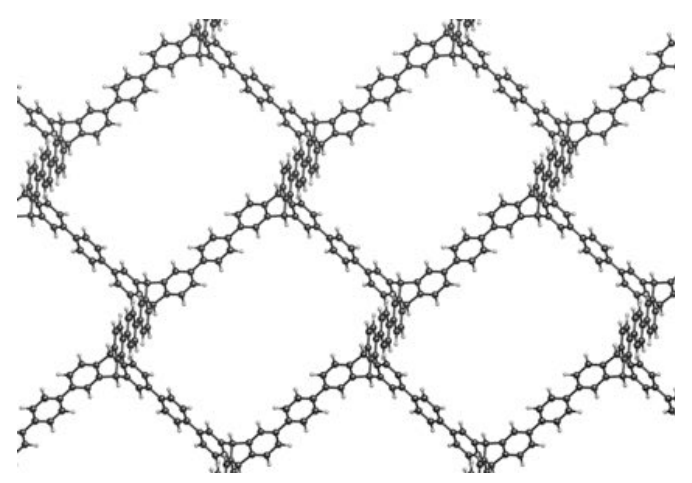

(c)
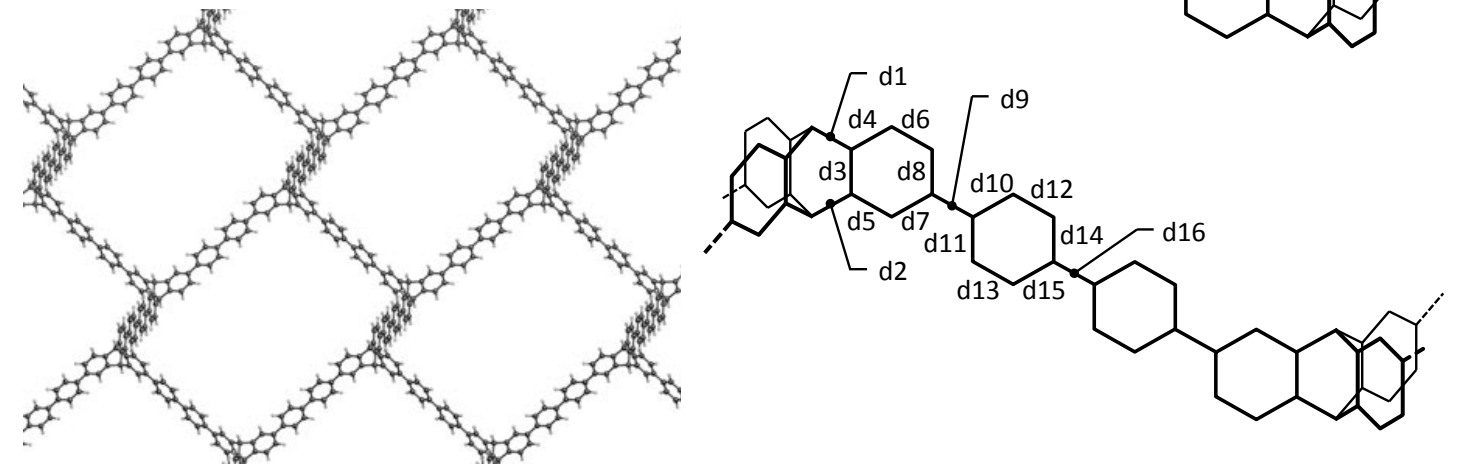

Fig.1. (Black and white) 


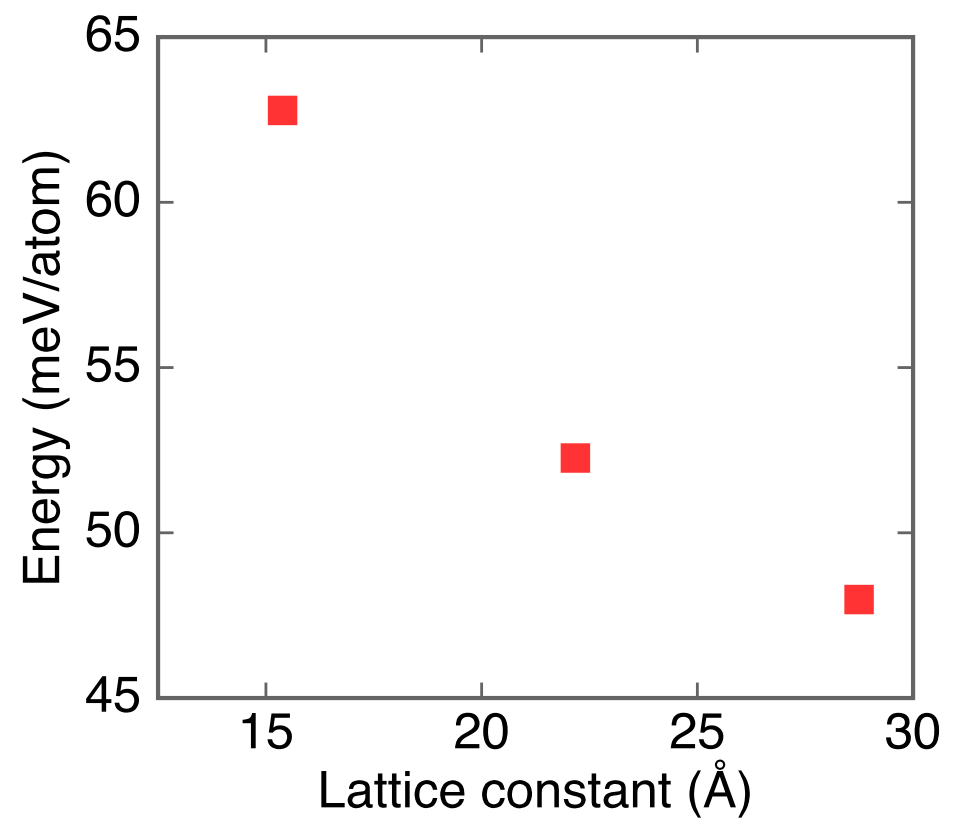

Fig. 2. (Color Online) 
(a)

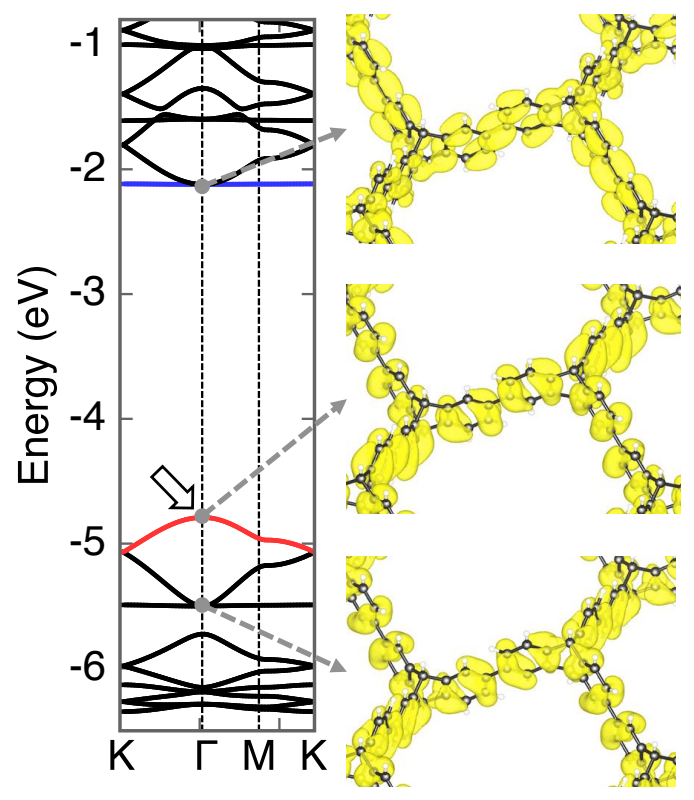

(c)

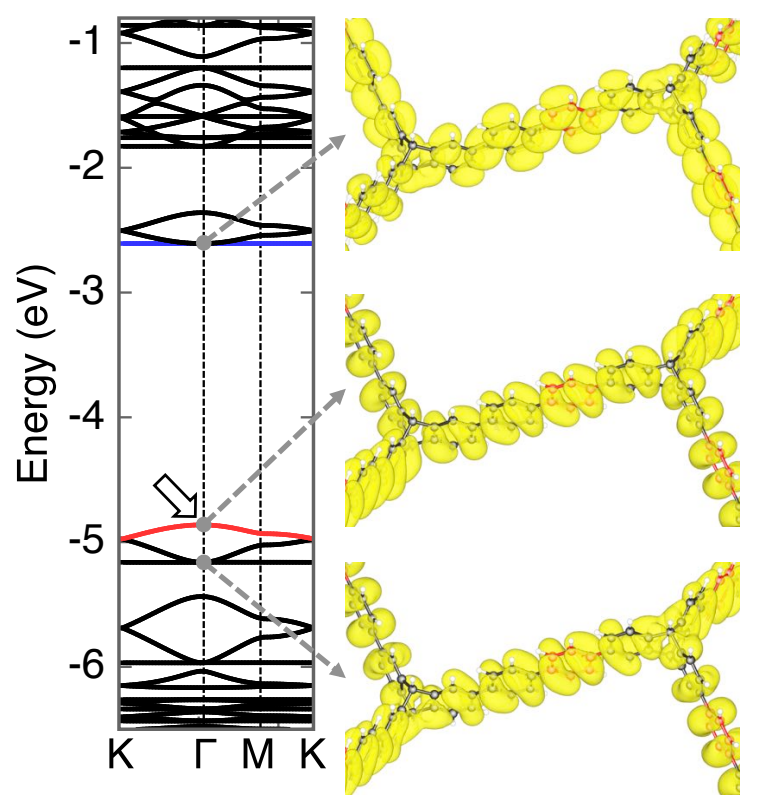

(b)

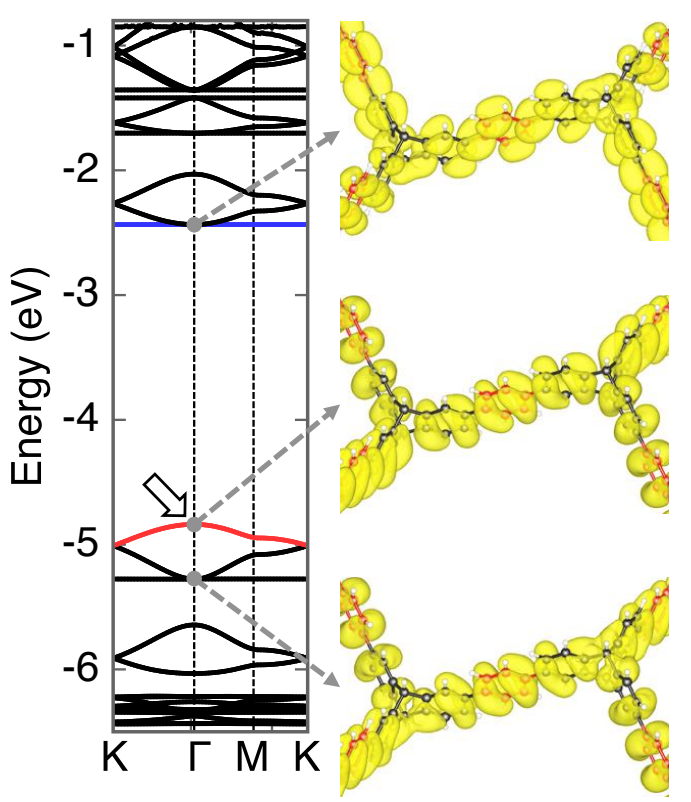

Fig. 3. (Color Online) 
(a)

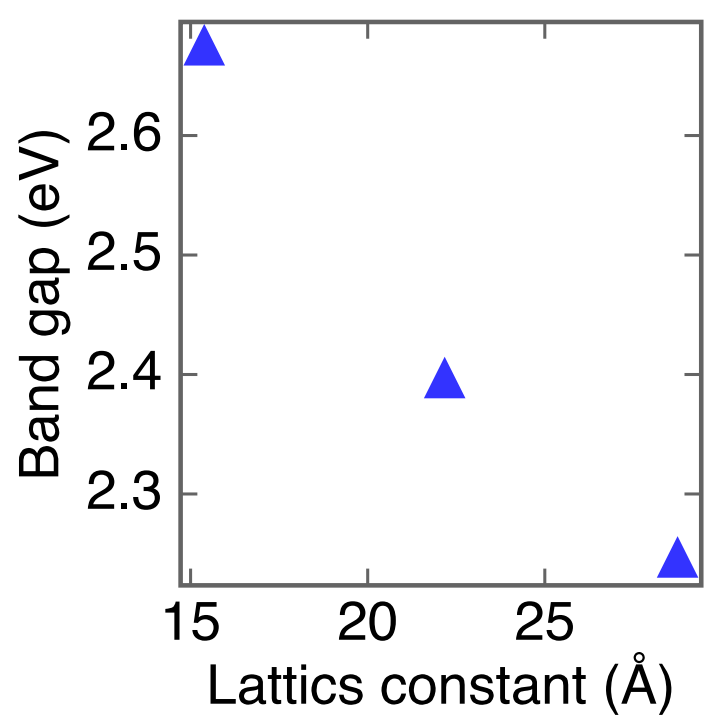

(c)

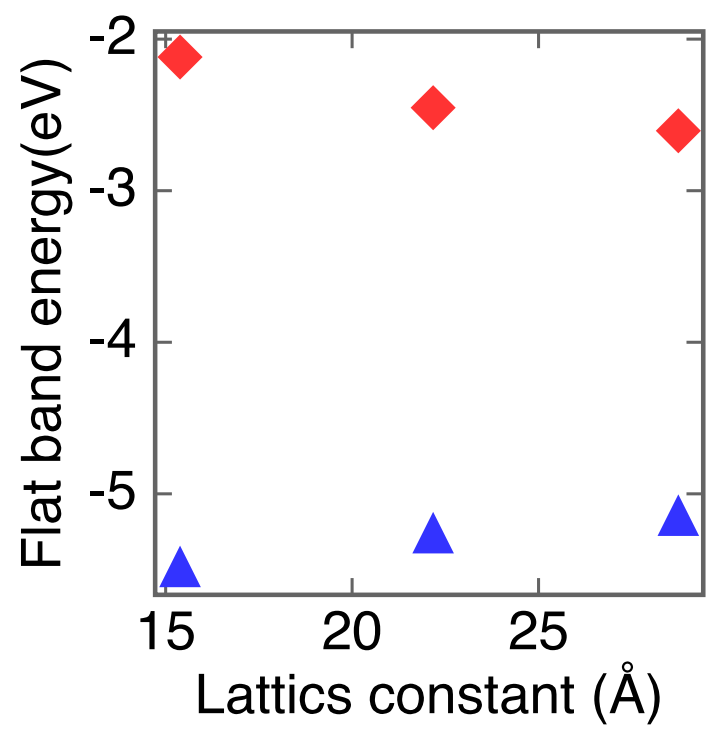

(b)

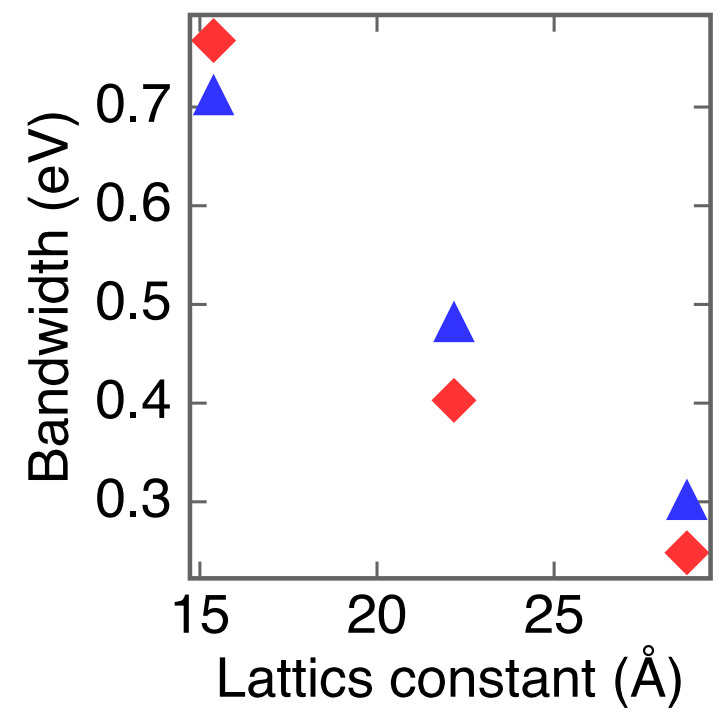

Fig. 4. (Color Online) 


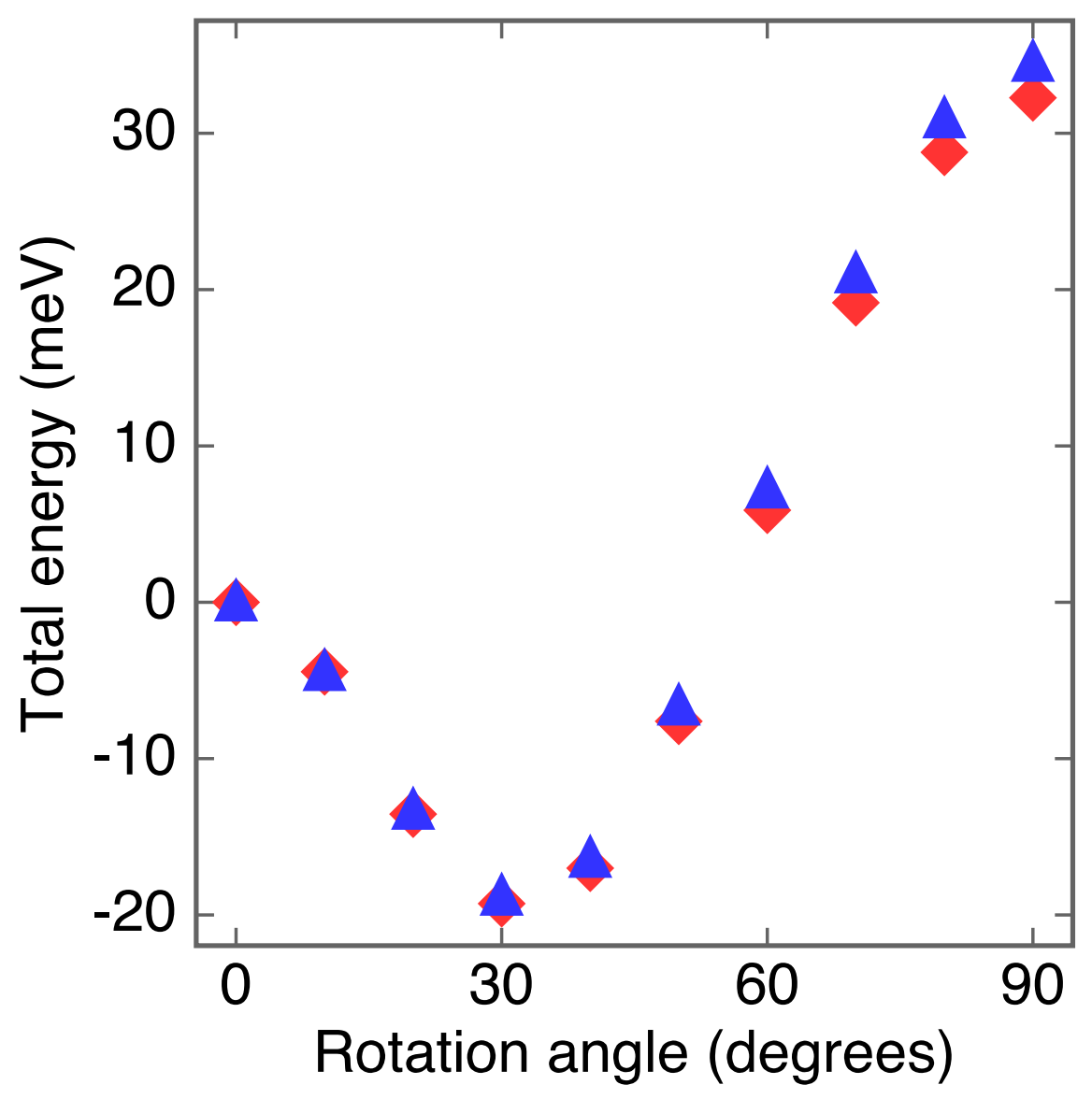

Fig. 5. (Color Online) 
(a)

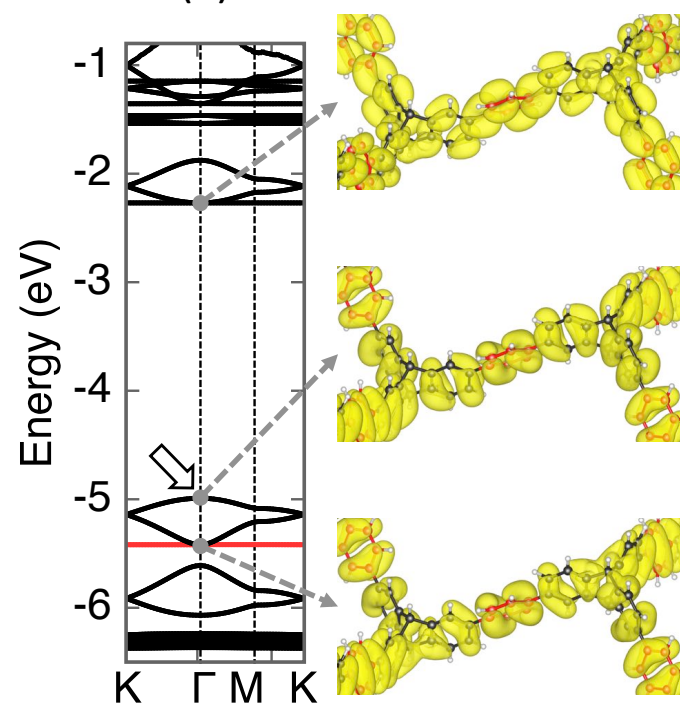

(c)

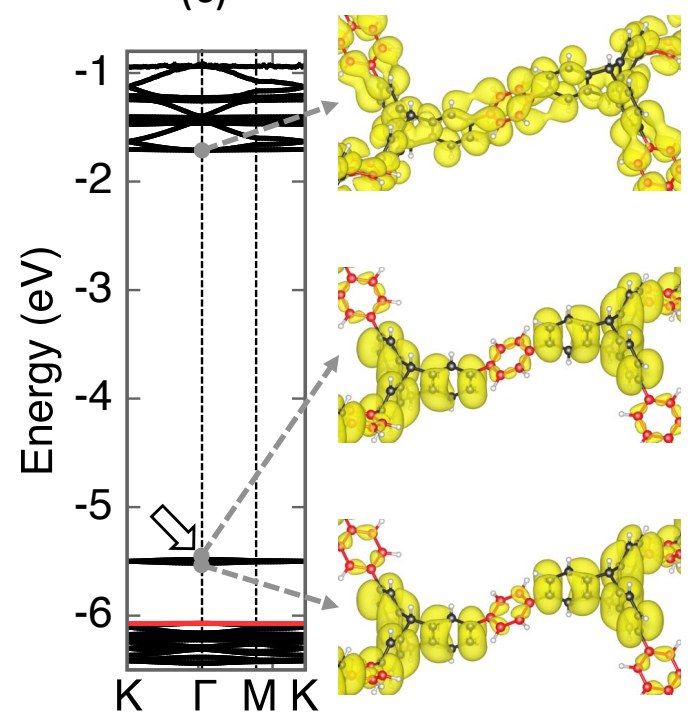

(b)

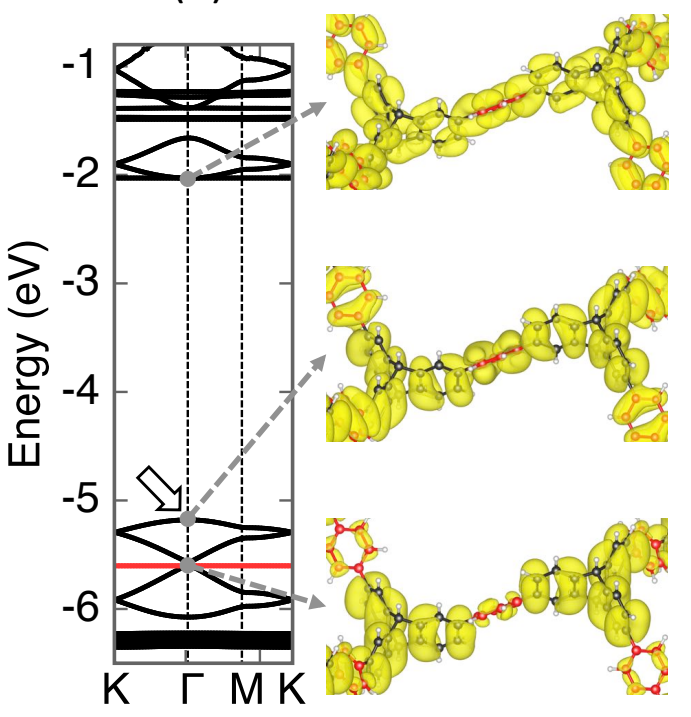

Fig. 6. (Color Online) 
(a)

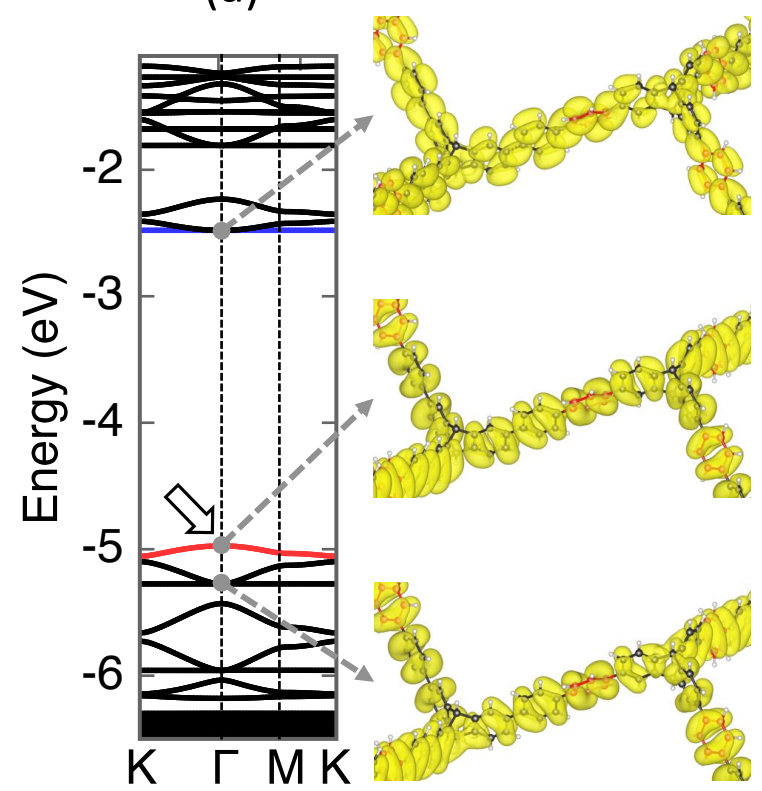

(c)

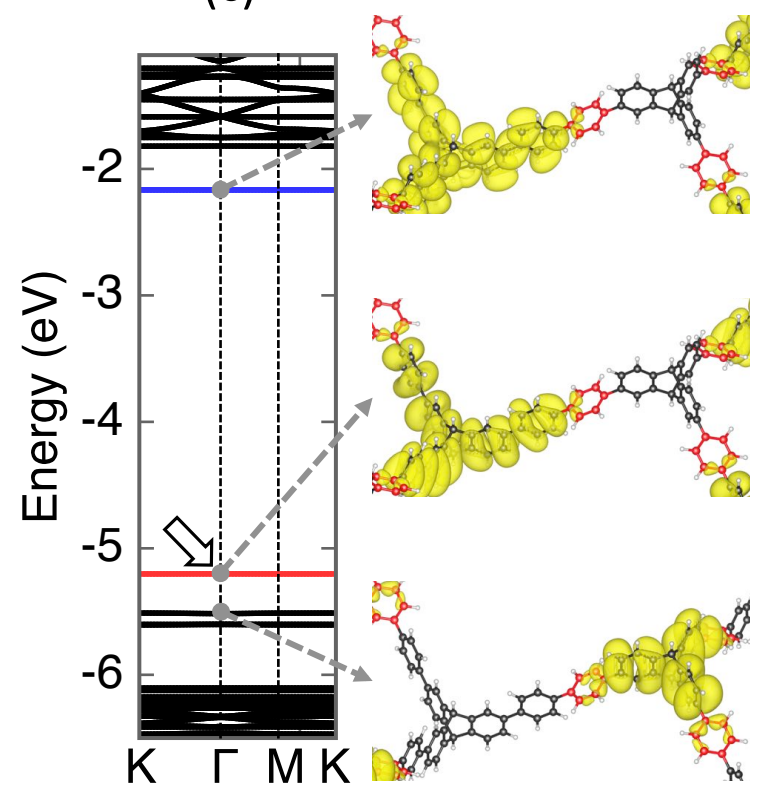

(b)

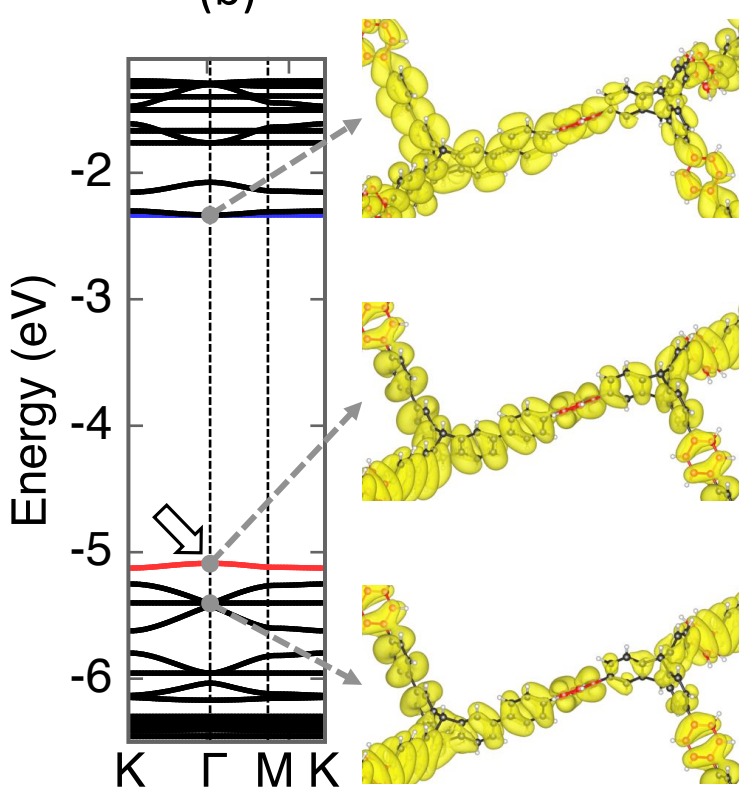

Fig. 7. (Color Online) 
(a)

$$
\frac{\left|t^{\prime}\right|}{t}=\infty \quad \frac{\left|t^{\prime}\right|}{t}=2 \quad \frac{\left|t^{\prime}\right|}{t}=1.75 \frac{\left|t^{\prime}\right|}{t}=1.5 \frac{\left|t^{\prime}\right|}{t}=1.25 \quad \frac{\left|t^{\prime}\right|}{t}=1
$$

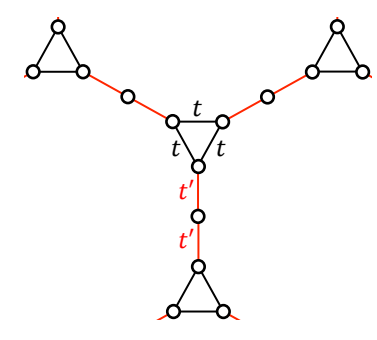
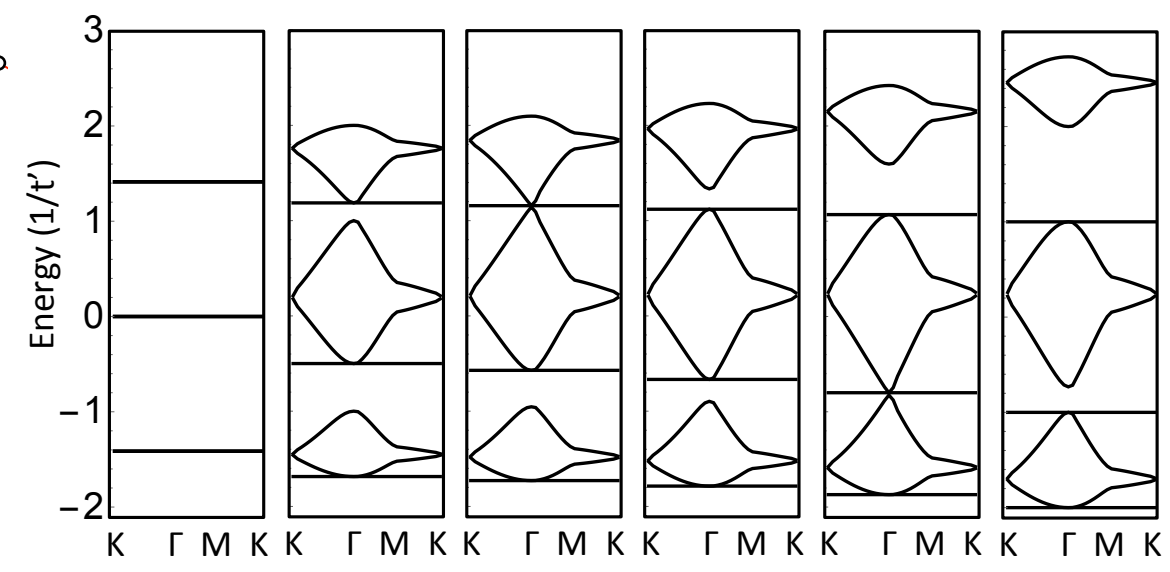

(b)

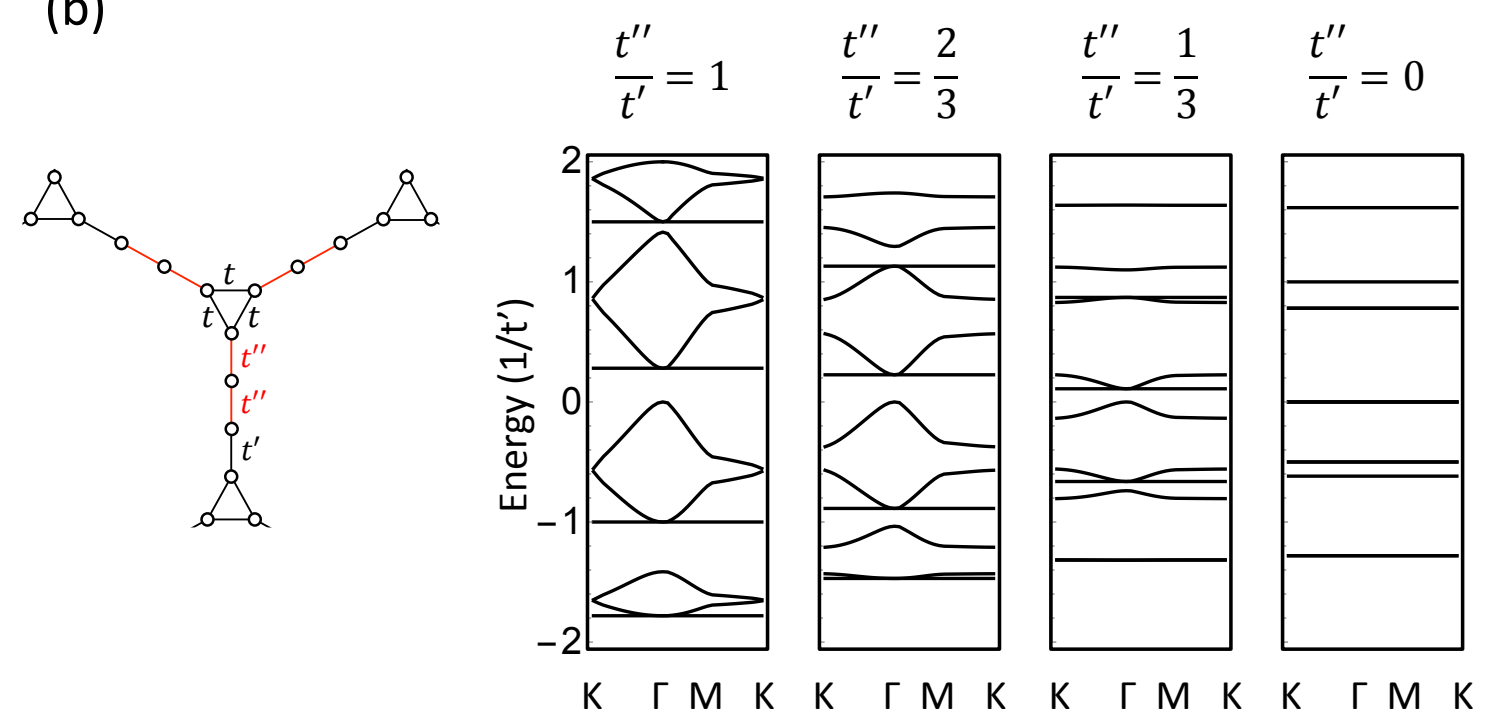

Fig. 8. (Color Online) 